\title{
A Rare Case of Unilateral Foix-Chavany-Marie Syndrome
}

\author{
Courtney Raab, Farrukh Chaudhry, MD \\ Community Hospital-Munster Department of Neurocritical Care, Indiana University School of Medicine
}

Background: Bilateral anterior opercular syndrome, or Foix-Chavany-Marie Syndrome (FCMS), is a neurological condition characterized by bilateral anterior opercular lesions. Common presenting symptoms of this rare suprabublar palsy include dysarthria, or slurred speech, as well as paralysis of facial, pharyngeal, lingual, and masticatory voluntary muscles while autonomic function is preserved. ${ }^{1}$ Most cases of FCMS are bilateral, yet some rare cases have been reported with unilateral opercular lesions with preexisting contralateral white matter lesions. ${ }^{2}$ In this case report we present a rare case of unilateral FCMS in a patient who had an acute anterior left opercular infarct, as well as a residual right parietal subcortical stroke.

Project Methods: The patient we present is a 49 year old African American female with a history of hypertension and previous stroke with residual spastic right hemiplegia who presented to the ED for evaluation of right sided facial droop, right sided weakness, and slurred speech. Patient was evaluated by acute stroke team and was treated with intravenous thrombolysis (Alteplase). On hospital day 2 , the patient developed complete disarticulation, unable to produce any speech but able to communicate by appropriate gestures and writing. The patient's symptoms included anarthria, weakness of bilateral masseters, and lateral/medial pterygoids without dysphagia.

Results: The MRI brain showed left insular region/frontal opercular ischemic stroke and a small right parietal subcortical ischemic stroke, likely embolic in nature. Over the hospital stay, the patient's motor function improved but her anarthria persisted. These radiologic findings along with the symptomology proved consistent with FCMS. She was discharged to home with an NIH stroke scale of 5 and recommended outpatient speech therapy.

Conclusion: In this case report, we describe a patient that presents with an extremely rare case of unilateral FCMS, with a preexisting contralateral parietal infarct that could have collectively caused anarthria and masseter weakness.

\section{Works Cited}

1. Milanlioglu A, Aydın MN, Gökgül A, Hamamcı M, Erkuzu MA, Tombul T. Ischemic Bilateral Opercular Syndrome. Case Reports in Medicine. 2013;2013:1-3. doi:10.1155/2013/513572.

2. Sa F, Cordeiro IM, Mestre S, Nzwalo H. Unilateral opercular infarction presenting with FoixChavany-Marie syndrome. Case Reports. 2014;2014. doi:10.1136/bcr-2014-206439. 